\title{
EFFECTIVENESS OF BLOCK ITERATIVE SCHEMES IN COMPUTING THE SEISMIC RESPONSE OF BUILDINGS WITH NONLINEAR BASE ISOLATION
}

\author{
A. H. Barbat, N. Molinares and R. Codina \\ Technical University of Catalonia, Edificio C1, Campus Norte UPC, Gran Capitan, s/n, \\ 08034 Barcelona, Spain
}

(Received 21 May 1994)

\begin{abstract}
The conventional form of solving the equations of motion governing the seismic response of building structures with nonlinear base isolation consists of using monolithic step by step integration methods. As an alternative, the application of a block iterative scheme is examined in this paper. After describing the particularities of the equations of motion of the structure with base isolation, different block iterative schemes are described. Their effectiveness is discussed and compared with that of monolithic step by step integration methods. Adequate examples are used with the aim of performing numerical comparisons.
\end{abstract}

\section{INTRODUCTION}

Base isolation systems partially uncouple a structure from the seismic ground motion by means of specially designed, replaceable, devices inserted between the structure and its foundation. These devices are capable of absorbing part of the energy induced by earthquakes [1-4] and drastically reduce the seismic action transmitted to the structure. A numerical simulation of their effect on the seismic response of structures requires algorithms capable of analysing structures with both elastomeric (hysteretic) bearings and sliding (frictional) bearings [5, 6]. Different numerical schemes for solving the equations of motion have been proposed. The most often used numerical procedures are monolithic step-by-step integration schemes, that is, schemes that lead to algebraic systems of equations involving both the degrees of freedom corresponding to the structure and the foundation. On the other hand, there is the possibility of coupling these two sets of unknowns iteratively, rather than by solving the full algebraic system. These iterative methods, when combined with the proper linearization of the nonlinear terms, yield block iterative schemes such as those considered in this paper. Their capability for solving other problems, such as the dam-fluid interaction or the motion of thermally driven flows, is described in Ref. [7]. In this paper, the application of schemes of this type in computing the seismic response of building structures with base isolation is considered, this being a problem of two systems coupled across their boundary conditions. The corresponding equations of motion are first written and then details concerning the possibilities of numerical computation of the seismic response are given. Different manners of formulating block itera- tive schemes are described in a generic form and are then applied to the studied case. Their effectiveness is then explored on the basis of a complete numerical example.

\section{EQUATIONS OF MOTION}

\subsection{Formulation}

In the following developments it is assumed that the isolated structure has a linear behaviour due to the effect of the base isolator. Also the bearings will be considered to be hysteretic, frictional, or a combination of both. Moreover, the base isolation system is assumed to have only one degree of freedom, the displacement $d_{\mathrm{b}}$ in the direction of the earthquake. Under these conditions the corresponding system of equations of motion for the building structure of Fig. 1 is

$$
\mathbf{M} \ddot{\mathbf{D}}+\mathbf{C D}+\mathbf{K D}=-\mathbf{M J}\left[\ddot{d}_{\mathrm{b}}+a(t)\right],
$$

where $\mathbf{D}$ is the displacement vector rclative to the base of the structure. The mass matrix $\mathbf{M}$, the damping matrix $\mathbf{C}$, the stiffness matrix $\mathbf{K}$ and the vector $\mathbf{J}$ which express the rigid body motion according to the degrees of freedom of the model, are dependent on the procedure used in the spatial discretization. In the particular case where the structure is modelled as a shear building, the matrix $\mathbf{M}$ is diagonal, $\mathbf{K}$ is tri-diagonal and $\mathbf{J}$ is a unit vector. The damping matrix $\mathbf{C}$ is considered to be of Rayleigh type and is computed using a direct modal evaluation method [8]. $\dot{d}_{\mathrm{b}}$ is the acceleration of the base relative to the ground, according to its single degree of freedom and $a(t)$ is the ground acceleration. System (1) does not contain the equation corresponding to the degree of freedom of 


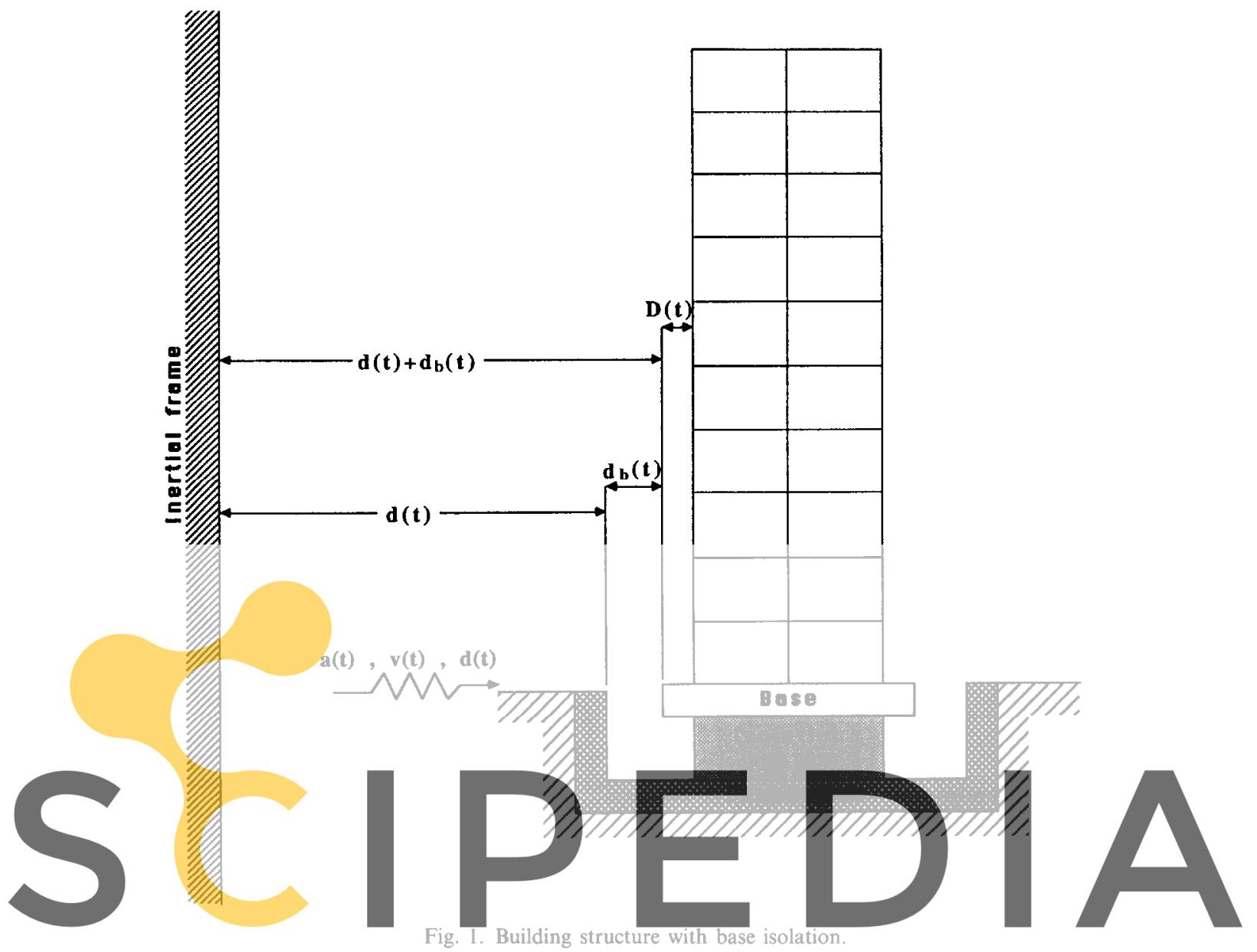

\section{Register for free at https//www.scipedia.com to
the base. The equation describing its motion can be} written as

$$
\begin{array}{r}
\mathbf{J}^{\mathbf{T}} \mathbf{M}\left[\ddot{\mathbf{D}}+\mathbf{J}\left(\ddot{d}_{\mathrm{b}}+a\right)\right]+m_{\mathrm{b}}\left(\ddot{d}_{\mathrm{b}}+a\right)+c_{\mathrm{b}} \ddot{d}_{\mathrm{b}} \\
+k_{\mathrm{b}} d_{\mathrm{b}}+f=0,
\end{array}
$$

where $f$ is the sum of the restoring force $f_{1}$ due to the hysteretic elements of the bearing and $f_{2}$ the force due to the frictional elements. An adequate mathematical description of these forces is required to solve the problem. $m_{\mathrm{b}}$ is the mass of the isolation system, $k_{\mathrm{b}}$ the stiffness of its complementary elastic device and $c_{\mathrm{b}}$ its damping coefficient.

\subsection{Computer simulation of the base isolators}

The constitutive models which describe the behaviour of the seismic base isolation system have to be able to consider [9]:

- the shear stiffness in hysteretic bearings before and after yielding;

- the loss of shear stiffness in hysteretic bearings as a function of the increment of the axial force;

- the variation of the friction coefficient as a function of the velocity and the pressure in frictional bearings. download the version without the watermark The hysteretic isolation devices will be formulated using Wen's constitutive model [10-12], which expresses the force $f_{1}$ as

$$
f_{1}=f^{y} z
$$

where $f^{y}$ is the yield force and $z$ an auxiliary variable expressed as a function of the displacement $d_{b}$ of the base relative to the ground and of its derivative $\dot{d}_{b}$. This variable is the solution of the differential equation

$$
\dot{z}=D \dot{d}_{\mathrm{b}}-v|z|^{n} \dot{d}_{\mathrm{b}}-\gamma|z|^{n-1} z\left|d_{\mathrm{b}}\right|
$$

The parameters $A, v, \gamma$ and $n$ allow a description of the hysteretic cycles for a wide range of materials from elastic to elasto-plastic ones.

For the frictional isolation devices, the calculation of the pure frictional force $f_{2}$ is made by using the equation [10]

$$
f_{2}=\mu Q \operatorname{sign} d_{\mathrm{b}},
$$

where $Q$ is the force perpendicular to the friction surface. The coefficient $\mu$ is calculated from the equation

$$
\mu=\mu_{\max }-\Delta \mu \mathrm{e}^{-\beta\left|\dot{d}_{b}\right|}
$$


where $\beta$ is a constant, $\mu_{\max }$ the fricitional coefficient for high sliding velocities and $\Delta \mu$ the difference between $\mu_{\max }$ and the frictional coefficient for slow sliding velocities, which is also considered to be a constant [10]. Whenever the base sticks to the foundation, the non-sliding condition

$$
\dot{d}_{\mathrm{b}}=0, \quad \dot{d}_{\mathrm{b}}=0, \quad d_{\mathrm{b}}=0
$$

has to be applied. If this condition is applied to eqn (2) then [12]

$$
\mu Q-\left|m_{\mathrm{b}} a+\mathbf{J}^{\mathrm{T}} \mathbf{M} \ddot{\mathbf{D}}+\mathbf{J}^{\mathrm{T}} \mathbf{M} \mathbf{J} a\right|>0 .
$$

Slip will occur if stick condition (8) fails and then eqn (2) has to be used.

\subsection{Criteria for selecting the solution procedure}

The type of problem defined by eqns (1) and (2) conditions the solution procedure that can be applied. This is because the force $f$ has both hysteretic and frictional components. The hysteretic component corresponds to a model, which for certain values of the parameters $A, v, \gamma$ and $n$ produces great energy absorption, so the problem is very nonlinear. The frictional component is characterized by a coefficient of friction that depends on the velocity and the additional stick condition

dition, all this is complica

isolation system has stops th cements. From the poin
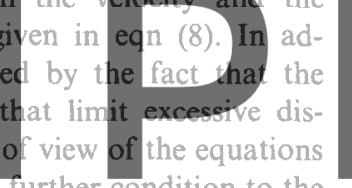

of motion, these stops add a further condition to the treatment of the non-linearity. During the time

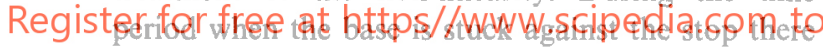
is no interaction. Obviously, in a well designed isolation device, these stops are not reached for a seismic ground motion within the design range. Nevertheless, the structure-base system is difficult to analyse and any numerical procedure requires the use of very small time increments [12]. Step-by-step monolithic integration procedures have been applied in all the consulted references [2-12]. They all have the disadvantage of requiring a large number of iterations as their convergence process is very slow. This paper considers an alternative method of block iteration to reduce the number of iterations and improve convergence $[7,13]$. An efficiency study is made by comparing the block iteration scheme to the monolithic scheme which treats non-linearity as an iterative actualization of the force $f$ in eqn (2). Both methods are combined with two discretizations in time: Newmark's simple step method and the two-step central difference scheme.

\section{BLOCK ITERATIVE SCHEMES}

\subsection{General theory}

This section explains the general method of block iteration to solve equations that describe the behaviour of coupled systems through their boundary conditions. The algorithm requires a certain discretization procedure for the equations of motion and solves them in a single iterative loop that considers their linearization and coupling [7, 13].

The equations that describe a generic coupled problem of the type studied in this paper may be reduced by the application of the discretization procedure to a non-linear algebraic system with the form [13]

$$
\left[\begin{array}{cc}
A_{11} & A_{12} \\
A_{21} & A_{22}(\mathbf{y})
\end{array}\right]\left[\begin{array}{l}
\mathbf{x} \\
\mathbf{y}
\end{array}\right]=\left[\begin{array}{l}
\mathbf{q}_{1} \\
\mathbf{q}_{2}
\end{array}\right]
$$

where $\mathbf{x}$ and $\mathbf{y}$ are the vectors to be determined, $\mathbf{q}_{1}$ and $\mathbf{q}_{2}$ are the force vectors and $\mathbf{A}_{i j}, i, j=1,2$ are matrices with $\mathbf{A}_{22}$ depending on $\mathbf{y}$. The equations of system (9) are coupled linearly. The matrix $\mathbf{A}_{22}$ is linearized in the following way:

$$
\mathrm{A}_{22}\left(\mathrm{y}^{(i)}\right) \mathrm{y}^{(i)} \approx \mathrm{A}_{22}^{\mathrm{L}} \mathrm{y}^{(i)}+\psi\left(\mathrm{y}^{(i-3)}\right),
$$

where $\mathbf{A}_{22}^{L}$ is a linearized form of $\mathbf{A}_{22}$. Starting from eqn (9) the following monolithic form can be obtained:

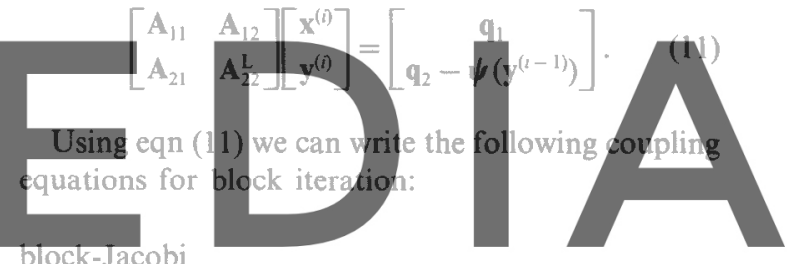

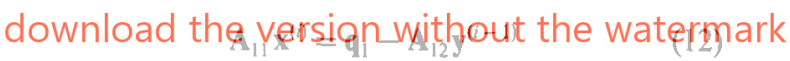

$$
\mathbf{A}_{22}^{L} \boldsymbol{y}^{(i)}=\mathbf{q}_{2}-\boldsymbol{\psi}\left(\mathbf{y}^{(i-1)}\right)-\mathbf{A}_{21} \mathbf{x}^{(i-1)} .
$$

This represents a first approach for implementing the block iteration procedure. Equation (12) is solved first to give a value for $\mathbf{x}^{(i)}$ and this is then used to solve eqn (13) to give the vector $y^{(n)}$. A second way of solving the problem [eqn (11)] considers the following way of expressing the equations:

\section{block-Gauss-Seidel}

$$
\begin{gathered}
\mathbf{A}_{11} \mathbf{x}^{(i)}=\mathbf{q}_{1}-\mathbf{A}_{12} \mathbf{y}^{(i-1)} \\
\mathbf{A}_{22}^{\mathrm{L}} \mathbf{y}^{(i)}=\mathbf{q}_{2}-\psi\left(\mathbf{y}^{(i-1)}\right)-\mathbf{A}_{21} \mathbf{x}^{(i)}
\end{gathered}
$$

or

$$
\begin{gathered}
\mathbf{A}_{22}^{\mathrm{L}} \mathbf{y}^{(i)}=\mathbf{q}_{2}-\psi\left(\mathbf{y}^{(i-1)}\right)-\mathbf{A}_{21} \mathbf{x}^{(i-1)} \\
\mathbf{A}_{11} \mathbf{x}^{(i)}=\mathbf{q}_{1}-\mathbf{A}_{12} \mathbf{y}^{(i)} .
\end{gathered}
$$

In the case of eqn (14), $x^{(i)}$ is first calculated from eqn (14a) and used to solve eqn (14b). In eqn (15), $y^{(i)}$ is calculated from eqn (15a) and then used to solve eqn (15b). 
The Jacobi-block procedure is now applied to perform the analysis of structures with base isolation.

\subsection{Time discretization}

As mentioned earlier, in order to develop a block iteration scheme, there must be a discretization of time in the equations of motion. Two types of formulation are considered. One uses the Newmark discretization for the velocity and the acceleration [14]

$$
\begin{aligned}
\ddot{\mathbf{D}}_{i+1}=\frac{1}{\beta \Delta t^{2}}\left[\mathbf{D}_{i+1}-\mathbf{D}_{i}-\dot{\mathbf{D}}_{i} \Delta t\right]-\left(1-\frac{\gamma}{2 \beta}\right) \ddot{\mathbf{D}}_{i} \\
\dot{\mathbf{D}}_{i} \mathbf{i}+1=\frac{\gamma}{\beta \Delta t}\left(\mathbf{D}_{i+1}-\mathbf{D}_{i}\right) \\
+\left(1-\frac{\gamma}{\beta}\right) \dot{\mathbf{D}}_{i}+\left(1-\frac{\gamma}{2 \beta}\right) \Delta t \ddot{\mathbf{D}}_{i}
\end{aligned}
$$

and the other is based on a formulation of central differences [14]

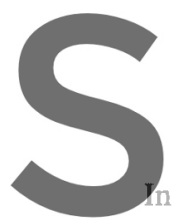

$$
\ddot{\mathbf{D}}_{i}=\frac{1}{\Delta t^{2}}\left[\mathbf{D}_{i+1}-2 \mathbf{D}_{i}+\mathbf{D}_{i-1}\right]
$$

step considered.

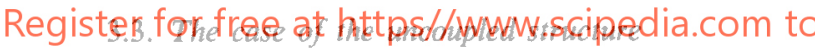

To uncouple eqn (I) using the first $q$ modes of vibration, the displacement vector is approximated as

$$
\mathrm{D}(t) \approx \sum_{i=1}^{q} \boldsymbol{\varphi}_{i} y_{i}(t)
$$

where $\varphi_{i}$ is the eigenvector and the function $y_{i}(t)$ is the general coordinate corresponding to the mode of vibration $i$. An equation of the uncoupled system has the following form [14]:

$$
\begin{aligned}
\ddot{y}_{j}(t)+2 v_{j} \omega_{j} \dot{y}_{j}(t)+\omega_{j}^{2} y_{j}(t) \\
=-\frac{\boldsymbol{\varphi}_{j}^{\mathrm{T}} \mathbf{M J}}{\boldsymbol{\varphi}_{j}^{\mathrm{T}} \mathbf{M} \boldsymbol{\varphi}_{j}}\left[\ddot{d}_{\mathrm{b}}+a(t)\right] ; \quad j=1,2, \ldots q,
\end{aligned}
$$

where $v_{j}$ and $\omega_{j}$ are the damping ratio and the eigenfrequency corresponding to the mode of vibration $j$. Transformation (20) is also applied to the variable $\mathbf{b}$ of eqn (2), giving

$$
\begin{array}{r}
\mathbf{J}^{\mathbf{T}} \mathbf{M}\left[\sum_{i=1}^{q} \varphi_{i} y_{i}(t)+\mathbf{J}\left(\ddot{d}_{\mathrm{b}}+a\right)\right]+m_{\mathrm{b}}\left(\ddot{d}_{\mathrm{b}}+a\right) \\
+c_{\mathrm{b}} d_{\mathrm{b}}+k_{\mathrm{b}} d_{\mathrm{b}}+f=0 .
\end{array}
$$

In this way the system to be solved is made of $q$ equations of type (21) and eqn (22). These equations may be expressed in the following compact form:

$$
\begin{array}{r}
\ddot{\mathbf{y}}+2 \boldsymbol{w} \omega \dot{\mathbf{y}}+\omega^{2} \mathbf{y}+\frac{\Phi^{\mathrm{T}} \mathbf{M} \mathbf{J}}{\Phi^{\mathrm{T}} \mathbf{M} \boldsymbol{\Phi}} \ddot{d}_{\mathrm{b}}--\frac{\Phi^{\mathrm{T}} \mathbf{M J}}{\boldsymbol{\Phi}^{\mathrm{T}} \mathbf{M} \boldsymbol{\Phi}} a(t) \\
\mathbf{J}^{\mathrm{T}} \mathbf{M} \boldsymbol{\Phi} \ddot{\mathbf{y}}+\left(\mathbf{J}^{\mathrm{T}} \mathbf{M J}+m_{\mathrm{b}}\right) \ddot{d}_{\mathrm{b}}+c_{\mathrm{b}} \ddot{d}_{\mathrm{b}}+k_{\mathrm{b}} d_{\mathrm{b}}+f \\
=-\left(\mathbf{J}^{\mathrm{T}} \mathbf{M} \mathbf{J}\right) a(t)-m_{\mathrm{b}} a(t),
\end{array}
$$

where $\Phi$ is the modal matrix corresponding to the first $q$ modes of vibration with dimensions $(n \times q), v$ is the diagonal matrix of damping ratios, $\omega$ is the diagonal matrix of frequency and $\omega^{2}$ the diagonal matrix of the squared frequencies. The terms $y_{i}$ and $\dot{y}_{i}$ in eqn (22) and $d_{\mathrm{b}}$ and $\dot{d}_{\mathrm{b}}$ in eqn (23) can be expressed in the function of the accelerations $\ddot{y}_{t}$ and $\ddot{d}_{b}$ by applying the Newmark discretization or that of central differences. A problem with the same characteristics as that described by the system of eqn (9) is thus produced. The procedure to be implemented is therefore the same as that described earlier.

\subsection{The case of the structure without uncoupling}

A similar formulation is used for the case of when

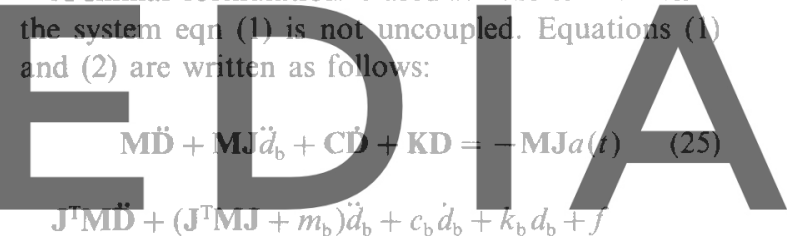

$J^{\mathrm{T}} \mathbf{M} \boldsymbol{D}+\left(J^{\mathrm{T}} \mathbf{M J}+m_{\mathrm{b}}\right) d_{\mathrm{b}}+c_{\mathrm{b}} d_{\mathrm{b}}+k_{\mathrm{b}} d_{\mathrm{b}}+$

download the versionjwithpyt the wyatermark

If discretization is applied we obtain once again a system similar to eqn (9).

\section{STEP BY STEP INTEGRATION ALGORITHMS}

In previous studies, the seismic response of base isolated structures has been simulated by solving the system of equations composed of eqns (1) and (2) using step-by-step integration methods [2-12]. Generally speaking, such methods are applied in the following sequence of operations:

(1) If the modal uncoupling of eqns (1) and (2) is used, the first $q$ modes of vibration are calculated and the uncoupling performed, which produces eqns (23) and (24).

(2) The computational process is started by initializing the values of the displacement, velocity and acceleration responses of the structure.

(3) The hysteretic force in eqn (4) is solved numerically using the Runge-Kutta scheme of fourth order. The value of $z$ is thus obtained. A base displacement limit has to be considered for each type of bearing analysed.

(4) Newmark's or central difference step-by-step integration methods are used to solve the equations 


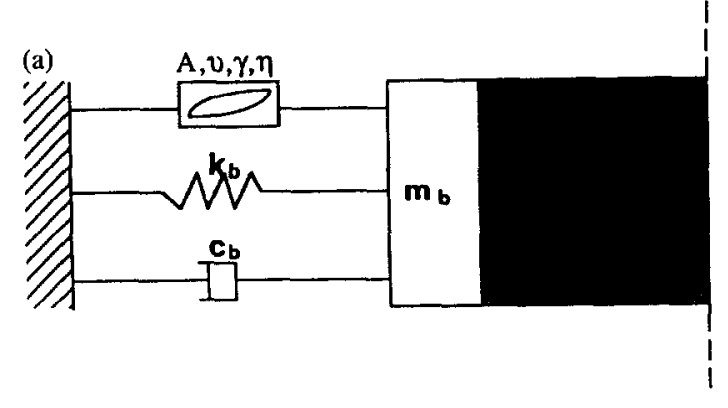

(1)
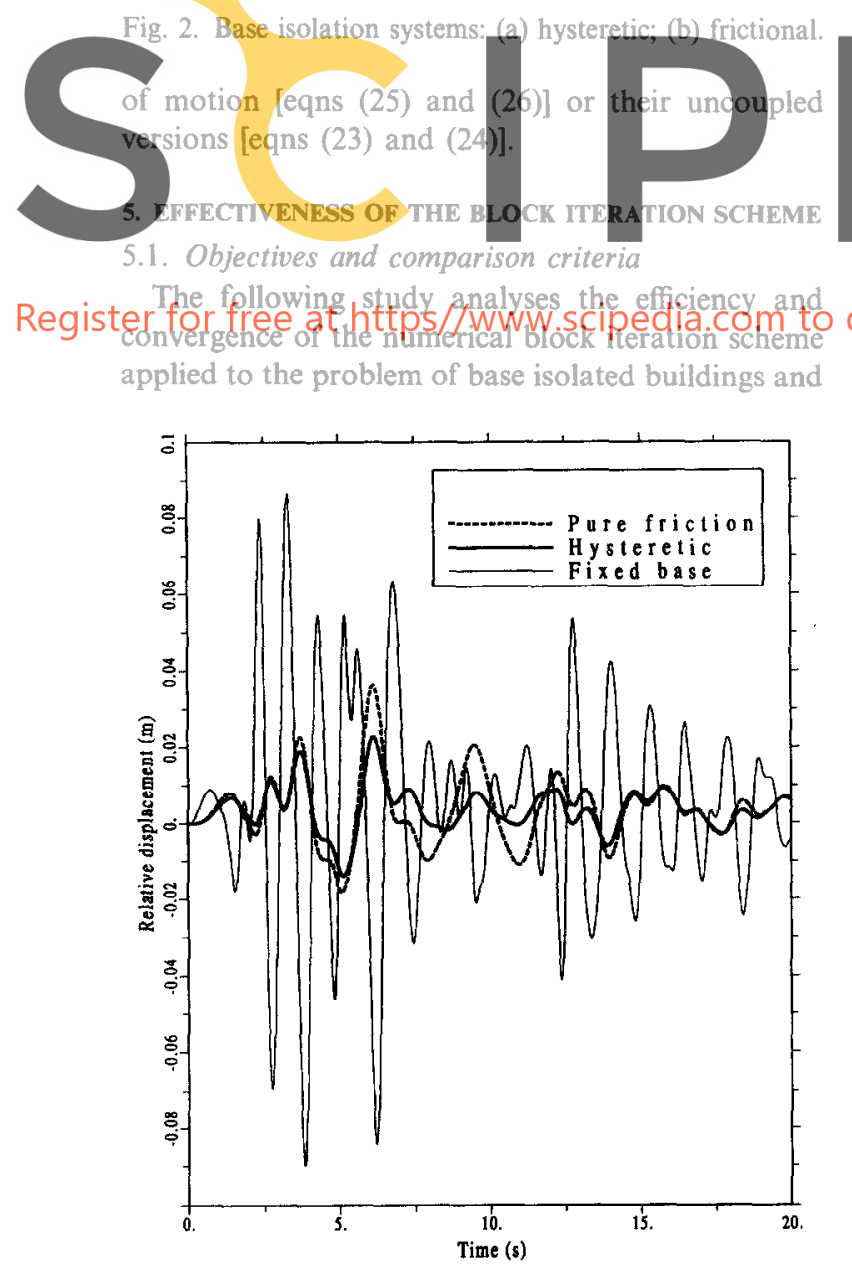

Fig. 3. Relative displacement history of the highest point of the structure.

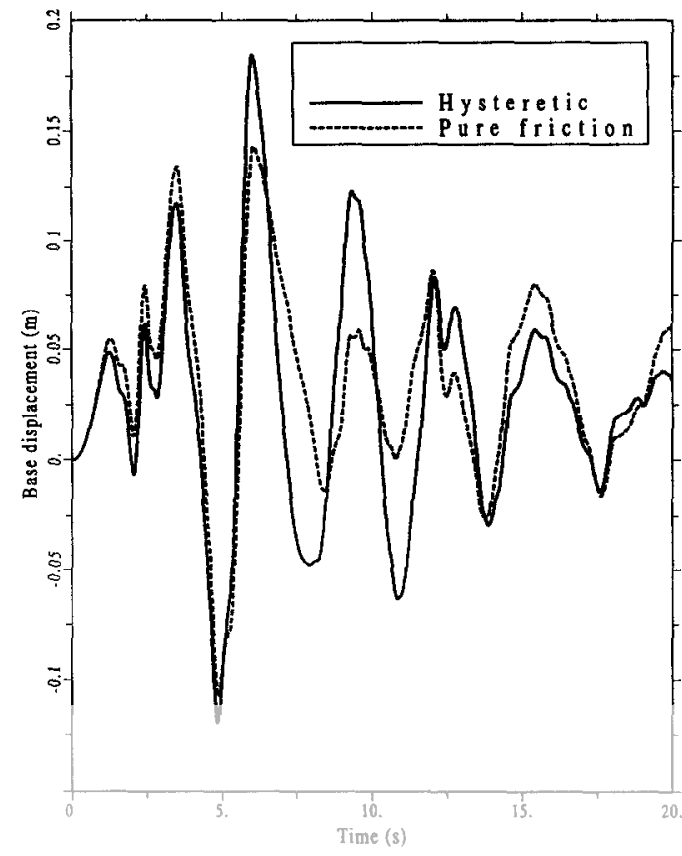

Fig. 4. Displacement history of the base relative to the foundation.

compares it to the Newmark monolithic integration method. This domparison is made using two different
methods of time discretization of the equations of
motion: Newmark differences and central differences.
The comparison between these two nethods uses the
number of iterations in each discretization instant and the variation of the residual norm at a given time instant.

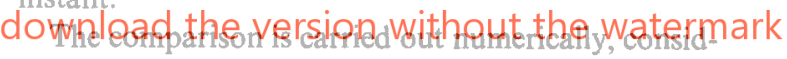
ering a shear building with 10 storeys and only one degree of frecdom in a horizontal direction (Fig. 1). The mass of each of the 10 storeys, as well as that of the base, is $6 \times 10^{5} \mathrm{~kg}$. The stiffness of the columns in the structure varies by $5 \times 10^{7} \mathrm{~N} \mathrm{~m}^{-1}$ between storeys from $9 \times 10^{8} \mathrm{~N} \mathrm{~m}^{-1}$ at the first level to $4.5 \times 10^{8} \mathrm{~N}$ $\mathrm{m}^{-1}$ at the top. The damping ratios have been fixed at 0.05 for all vibration modes.

Two types of base isolation were considered: hysteretic and frictional, as shown schematically in Fig. 2. In either of the two cases, the damping ratio of the bearing is 0.2 and its stiffness $k_{\mathrm{b}}=2 \times 10^{7} \mathrm{~N}$ $\mathrm{m}^{-1}$. For the aseismic hysteretic bearing, the secant stiffness required in eqn (2) is defined as a relation between the yield limit $f^{\gamma}$ and the yield displacement $d^{y}$ of the isolation. In the analysis $f^{y}$ was equal to $1.5 \times 10^{3} \mathrm{~N}$ and $d^{y}$ to $0.0245 \mathrm{~m}$. The constants defining the uniaxial hysteretic model are $A=1.0, v=0.5$, $\gamma=0.5$ and $n=1$. The characteristics of the frictional bearing are $\mu_{\max }=0.175, \mu_{\min }=0.100$ and $\beta=2$.

\subsection{Time history of the response}

The seismic excitation used in this case was an accelerogram of the El Centro (1940) earthquake. Figure 3 shows the time variation of the displacement 


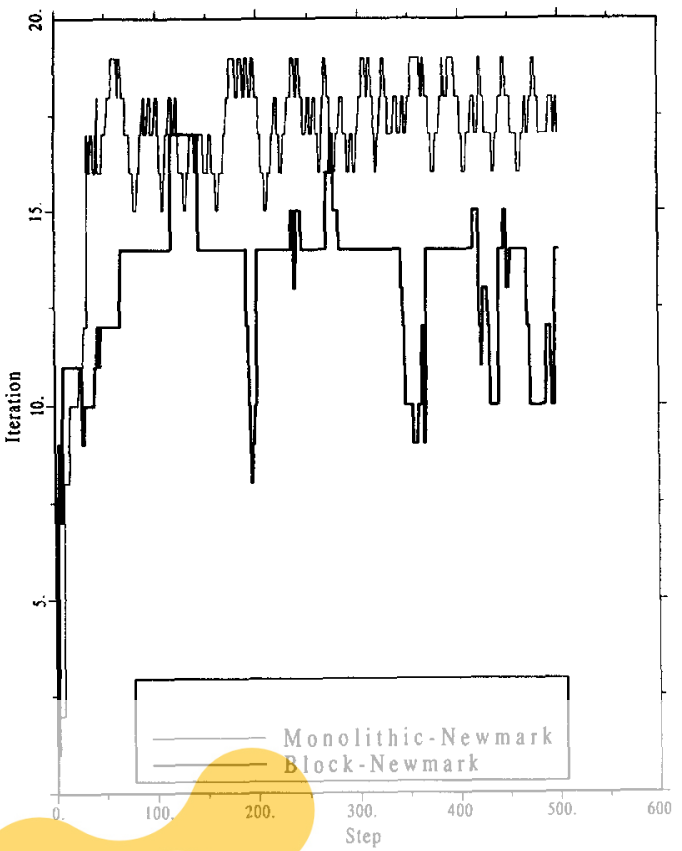

Fig. 5. Total number of iterations in each step, structure with modal uncoupling (Newmark method)

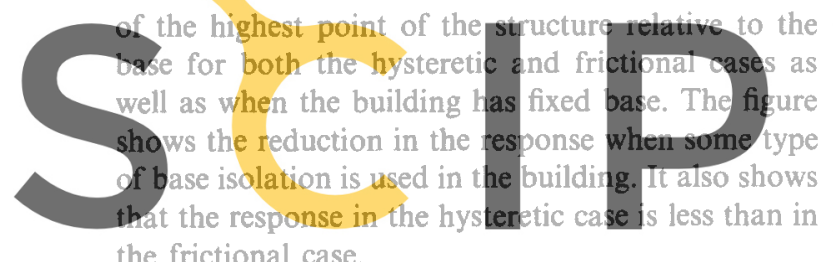

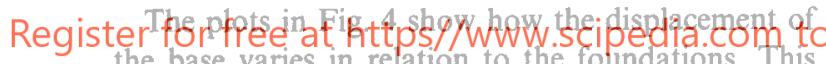

the base varies in relation to the foundations. This

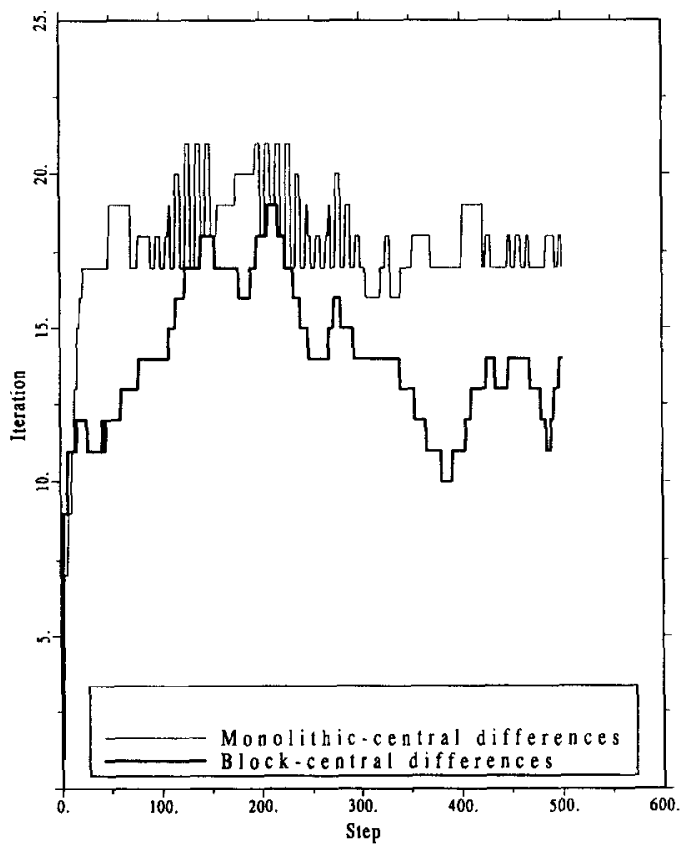

Fig. 6. Total number of iterations in each step, structure with modal uncoupling (central differences method).

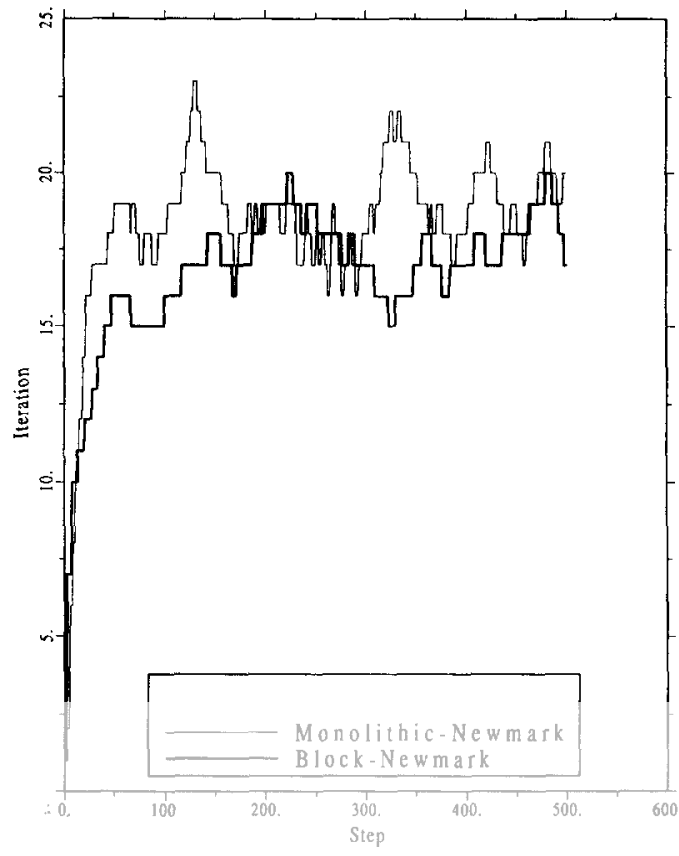

Fig. 7. Total number of iterations in each step, structure without modal uncoupling (Newmark method).

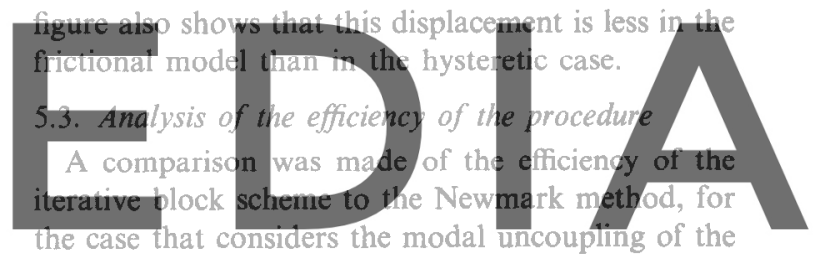

considers the modal uncoupling of the

system of equations of the structure (including the 10

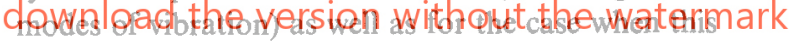

is not applied. The seismic excitation $a(t)$ has been

defined in this case as the sinusoidal acceleration $a(t)=A \sin \theta t$ with an amplitude $A$ of $3.5 \mathrm{~m} \mathrm{~s}^{-2}$ and a frequency $\theta$ of $10 \mathrm{rad} \mathrm{s}^{-1}$.

Figure 5 shows the results of the comparison between the Newmark method and the iterative block scheme using the Newmark discretization with a tolerance of $1 \%$. In both cases modal uncoupling of the structure was applied. The process of iterative blocks with Newmark discretization has a lower number of iterations throughout the calculation of the response of the system. Figure 6 shows the same comparison using central differences discretization. The iterative block scheme has a lower number of iterations than the monolithic solution method for each of the calculation steps.

In Figs 7 and 8 a comparison between the process of iterative blocks - using Newmark's and the central difference discretization--and the monolithic solution method can be seen for the case in which the modal uncoupling was not used. It can be observed that in both cases the block iteratives schemes are more efficient, as they require a smaller number of iterations to calculate the response.

All the above results correspond to a hysteretic base isolation system. Figures 9 and 10 show the same 


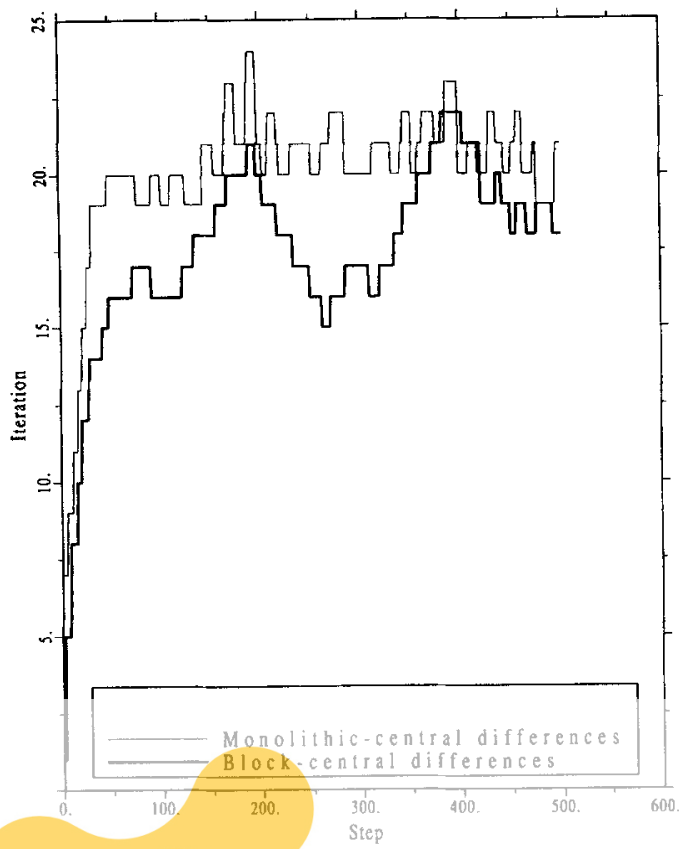

Fig. 8. Total number of iterations in each step, structure without modal uncoupling (central differences method).

comparison between the monolithic solution method

and the iterative block which uses the Niwmark

formulation, but for a fricional base isolation

The results of Fig. 9 correspond to the case of using

trior modal uncoupling, whereas Fig. 10 shows the

esults of the system of equations without uncou-

pling. Comparison of Figs 9 and 10 (frictional bear-

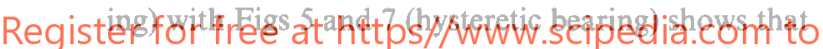

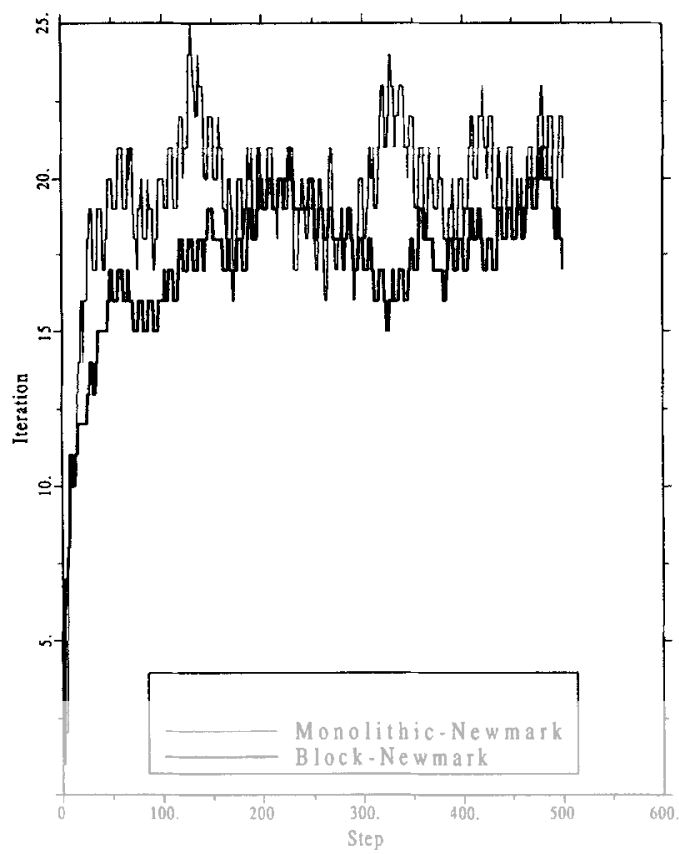

Fig. 10. Total number of iterations in each step, structure without modal uncoupling corresponding to frictional case (central differences method).

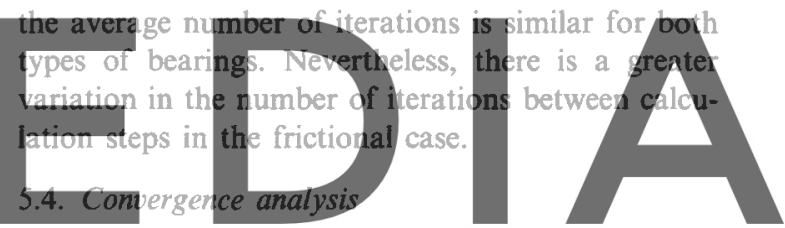

This section analyses the variation of the residual

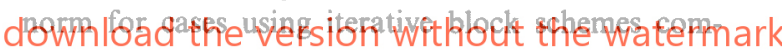

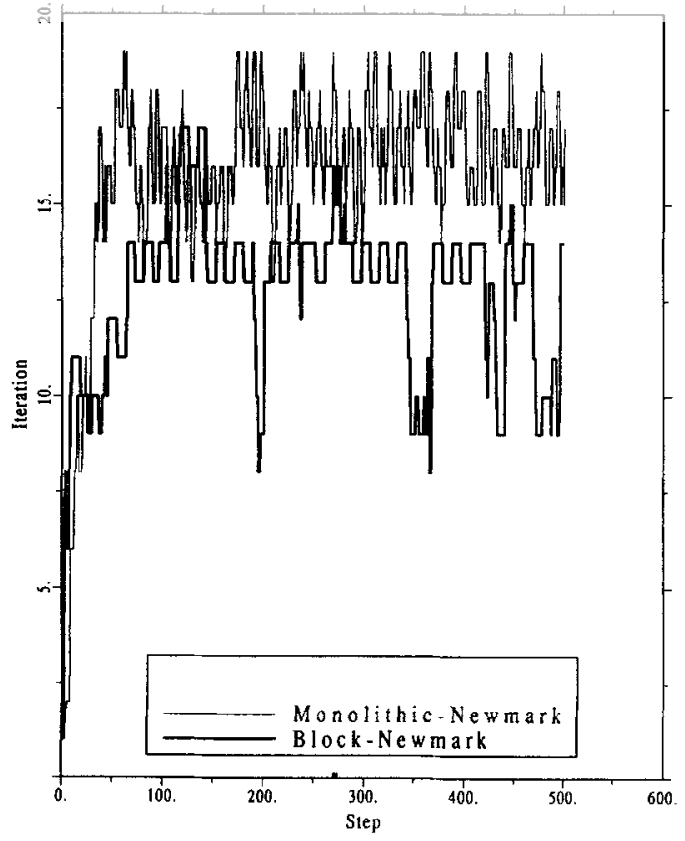

Fig. 9. Total number of iterations in each step, structure with modal uncoupling corresponding to frictional case (Newmark method).

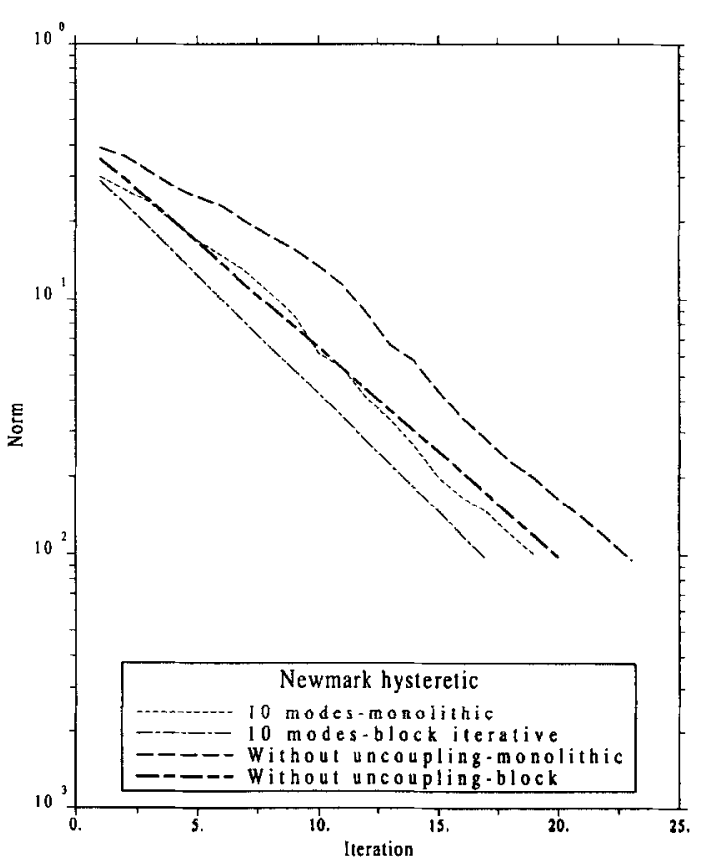

Fig. 11. Variation of the residual norm, structure with hysteretic isolation, Newmark method. 


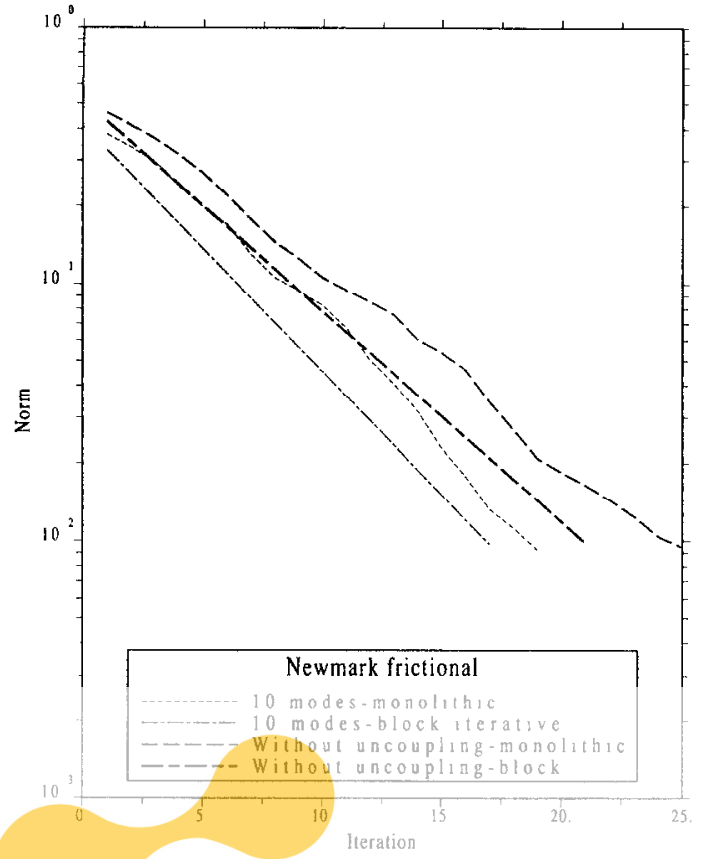

Fig. 12. Variation of the residual norm, structure with frictional isolation, Newmark method.

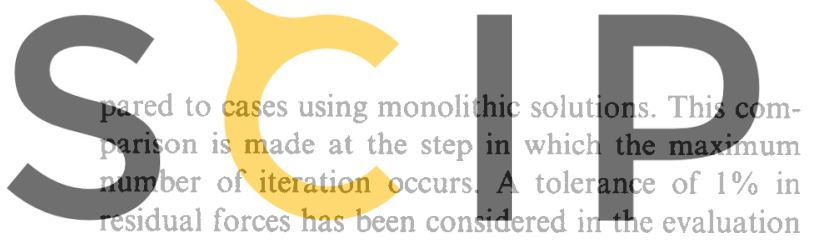

of the convergence of the iterative process. Figure 11

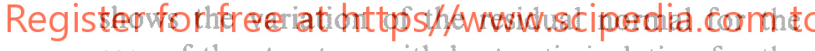
case of the structure with hysteretic isolation for the Newmark formulation, whereas Fig. 12 shows the solutions for the case of frictional isolation. The same comparison can be seen in Fig. 13 for the case of a hysteretic isolation, the central differences method being used. Note that the variation of the residual norm is similar in the two cases where the iterative block method is applied (Newmark and central differences).

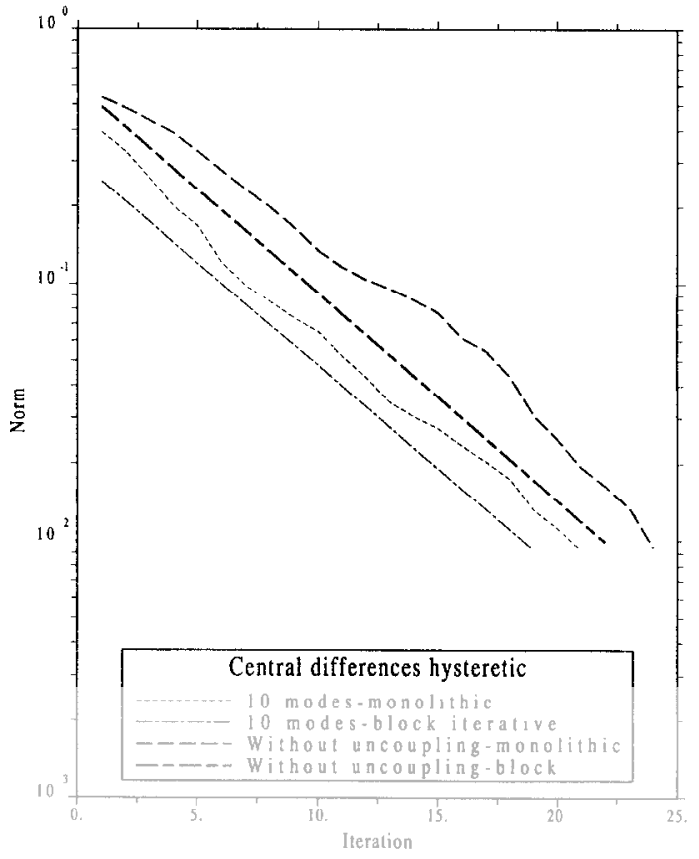

Fig. 13. Variation of the residual norm, structure with hysteretic isolation, central differences method.

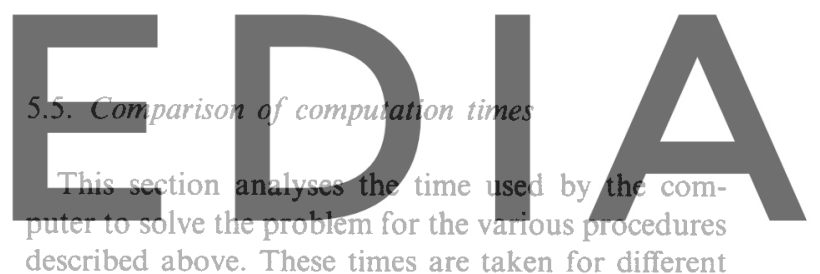

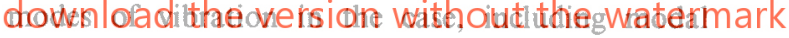
analysis and for the case without uncoupling, for both the monolithic scheme and that of iterative blocks. The following tables summarize the results obtained for the 10 storey building described earlier and for the sinusoidal vibration mentioned in Section 5.3. Table 1 shows the CPU time obtained in a VAX-6420 computer for the monolithic solution using the Newmark simple-step method and Table 2 shows the times corresponding to the block iterative

Table 1. Calculation times for the monolithic scheme

\begin{tabular}{crcc}
\hline Modes & Loops(s) & Iterations(s) & Systems solutions(s) \\
\hline 10 & 226.10621 & 0.02690 & 0.01221 \\
5 & 146.95659 & 0.01879 & 0.00586 \\
1 & 81.48294 & 0.01386 & 0.00210 \\
No uncoupling & 250.11798 & 0.02724 & 0.01180 \\
\hline
\end{tabular}

Table 2. Calculation times for the block iteration scheme

\begin{tabular}{crcc}
\hline Modes & Loops(s) & Iterations(s) & Systems solutions(s) \\
\hline 10 & 208.92214 & 0.02602 & 0.01124 \\
5 & 122.46383 & 0.01761 & 0.00481 \\
1 & 45.63047 & 0.01269 & 0.00175 \\
No uncoupling & 210.67212 & 0.02598 & 0.00967 \\
\hline
\end{tabular}


scheme using the Newmark discretization. These tables show that the block iterative schemes calculate the response of the system in less time than the monolithic scheme for all cases analyzed.

\section{CONCLUSIONS}

In this paper we have described the application of a block iterative technique to a nonlinear problem in structural analysis: the numerical simulation of the seismic response of structures with nonlinear base isolation. The method couples the equations describing the motion of the building with those of the isolated base. This coupling is done by means of an iterative procedure that accounts for the nonlinearity of the problem. This nonlinearity is particularly relevant for the type of isolation that we have considered, that is, frictional and hysteretic

In contrast to what happens when the monolithic system of equations is linearized, the block iterative technique treats the nonlinearity together with the coupling. This, apart from reducing the size of the problem by splitting it into two subproblems, may improve the convergence behaviour of the iterative scheme.

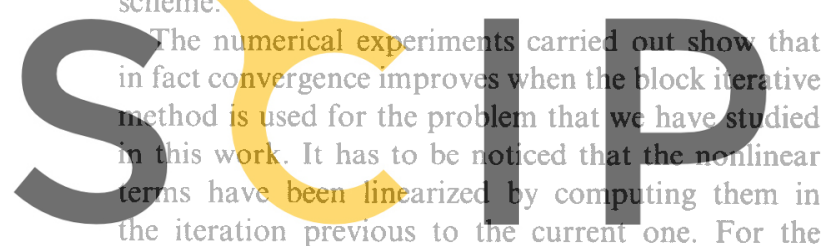

block iterative scheme, the resulting algorithm has a

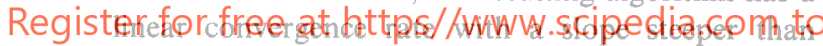

using the monolithic one and more uniform. Moreover, due to the smaller size of the subproblems to the dealt with, the computer time per iteration is also smaller.

Besides the better convergence and the lower computer cost, another aspect that makes the block iteralive technique attractive is the ease for its numerical implementation in existing codes. For the problem treated in this paper, codes dealing with fixed base structures are easily adapted simply by adding the equations corresponding to the isolation system, no further modification of the construction of the matrices and solution of the equations for the building being required.

\section{REFERENCES}

1. J. M. Kelly, Aseismic base isolation: review and bibliography. Soil Dyn. Earthquake Engng 5, 202-216 (1986).

2. R. I. Skinner, W. H. Robinson and G. H. McVerry, An Introduction to Seismic Isolation. Wiley, Chichester (1993).

3. I. G. Buckle and R. L. Mayes, Seismic isolation: history, application and performance-a world view. Earthquake Spectra 6, 161-202 (1990).

4. L. Jane and A. H. Barbat, Estructuras de edificación con aislamiento antisísmico. Centro Internacional de Métodos Numéricos en Ingeniería. Monogratia CIMNE-13 (1992).

5. Structural Engineering Association of California, Tentative gencral requircments for the design and construction of seismic isolated structures. In: Recommended Lateral Force Requirements and Commentary, Appendix II. Blue Book, CA (1990).

6. D. Way and V. Jeng, NPAD-A computer program for the analysis of base isolated structures. ASME Pressure Vessels and Piping Cont. Vol. PVP-147, pp. 65 94, Pennsylvania (1988).

7. M. Cervera, R. Codina and M. Galindo, On the computational efficiency and implementation of block-iterative algorithms for nonlinear coupled problems. Engineering Computations. (in press) (1995).

8. R. W. Clough and J. Penzien, Dynamics of Structures. McGraw-Hill, New York (1993)

9. S. Nagarajaiah, A. M. Reinhorn and M. C. Constantinou, 3D-BASIS - nonlinear dynamic analysis of three-

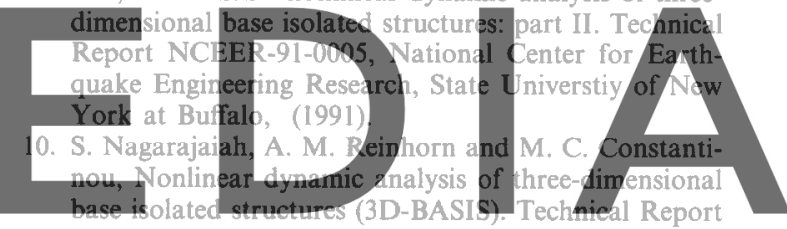
NCEER-89-0019, National Center for Earthquake Engineering Research State Winversity of New Yark at

11. P. E. Pinto and I. Vanzi, Base-isolation: reliability for different design criteria. In: Proc. Tenth World Conf. on Earthquake Engineering, Vol. 4, pp. 2033-2038, Madrid (1992).

12. L. Su, G. Ahmadi and I. G. Tadjbakhsh, A comparative study of performances of various base isolation systems, Part I: shear beam structures. Earthquake Engng struct. Dyn. 18, 11-32 (1989).

13. R. Codina, A finite element model for incompressible flow problems, Ph.D. thesis, Universidad Politécnica de Cataluña, Barcelona (1992).

14. J. M. Canet and A. H. Barbat, Estructuras Sometidas a Acciones Sísmicas-Cálculo por ordenador. Centro Internacional de Métodos Numéricos en Ingeniería, Barcelona (1988).

15. Y.-K. Wen. Method for random vibration of hysteretic system. J. Engng Mech. Div. ASCE 102, 249-263 (1976). 